\title{
Aircraft Control System Using Model Predictive Controller
}

\author{
Labane Chrif* ${ }^{1}$, Zemalache Meguenni Kadda ${ }^{2}$ \\ ${ }^{1}$ Labane chrif University of saida, Department of electrotechnic,Saida 20000, Algeria \\ ${ }^{2}$ LDEE Laboratory USTO, MB, Department of Automatic, Oran 31000, Algeria \\ ${ }^{*}$ Corresponding author, email: c_labane@hotmail.fr
}

\begin{abstract}
This paper concerns the application of model-based predictive control to the longitudinal and lateral mode of an aircraft in a terrain following task. The predictive control approach was based on a quadratic cost function and a linear state space prediction model with input and state constraints. The optimal control was obtained as the solution of a quadratic programming problem defined over a receding horizon. Closed-loop simulations were carried out by using the linear aircraft model. This project thesis provides a brief overview of Model Predictive Control (MPC). A brief history of industrial model predictive control technology has been presented first followed by a some concepts like the receding horizon, moves etc. which form the basis of the MPC. It follows the Optimization problem which ultimately leads to the description of the Dynamic Matrix Control (DMC). The MPC presented in this report is based on DMC. After this the application summary and the limitations of the existing technology has been discussed and the next generation MPC, with an emphasis on potential business and research opportunities has been reviewed. Finally in the last part we generate Matlab code to implement basic model predictive controller and introduce noise into the model.
\end{abstract}

Keyword: aircraft motion, flight control, lateral and longitudinal stability, model predictive control, optimization

Copyright @ 2015 Institute of Advanced Engineering and Science. All rights reserved.

\section{Introduction}

Originally developed to meet the specialized control needs of power plants and petroleum refineries, MPC technology can now be found in a wide variety of application areas including chemicals, food processing, automotive, and aerospace applications its reason for success is many, like it handles multivariable control problems naturally. But the most important reason for its success is its ability to handle constraints. Model predictive control (MPC) refers to a class of computer control algorithms that utilize an explicit process model to predict the future response of a plant. At each control interval an MPC algorithm attempts to optimize future plant behaviour by computing a sequence of future manipulated variable adjustments. The first input in the optimal sequence is then sent into the plant, and the entire calculation is repeated at subsequent control intervals. The basic MPC controller can be designed with proper restrictions on the prediction horizon and model length. The prediction horizon has to be kept sufficiently larger than control horizon. But after applying to many other applications we find as the complexity increases then we need techniques other than DMC like generalized predictive control (GPC) which are better. In modern processing plants the MPC controller is part of a multi-level hierarchy of control functions. It is often difficult to translate the control requirements at this level into an appropriate conventional control structure. In the MPC methodology this combination of blocks is replaced by a single MPC controller.

\subsection{Aircraft Control and Movement}

There are three primary ways for an aircraft to change its orientation relative to the passing air. Pitch (movement of the nose up or down), Roll (rotation around the longitudinal axis, that is, the axis which runs along the length of the aircraft) and Yaw (movement of the nose to left or right). Turning the aircraft (change of heading) requires the aircraft firstly to roll to achieve an angle of bank; when the desired change of heading has been accomplished the aircraft must again be rolled in the opposite direction to reduce the angle of bank to zero [7]. 


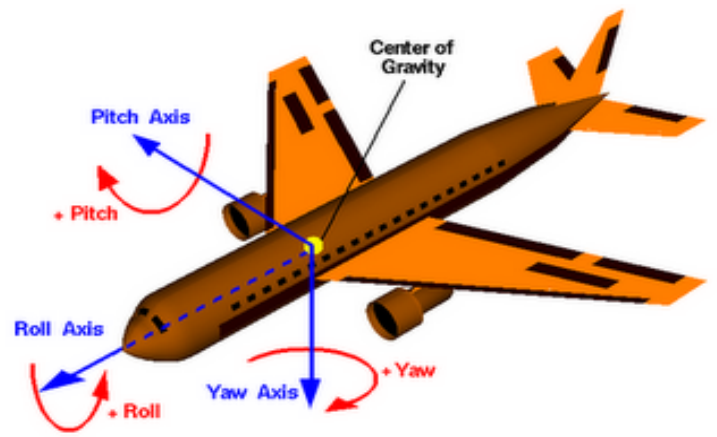

Figure 1. Axis of aircraft movements

\subsection{Flight Dynamics}

The science of air vehicle orientation and control there is three dimensions. The three critical flight dynamics parameters are the angles of rotation in three dimensions about the vehicle's center of mass, known as pitch, roll and yaw (quite different from their use as TaitBryan angles). Aerospace engineers develop control systems for a vehicle's orientation (attitude) about its center of mass. The control systems include actuators, which exert forces in various directions, and generate rotational forces or moments about the aerodynamic center of the aircraft, and thus rotate the aircraft in pitch, roll, or yaw. For example, a pitching moment is a vertical force applied at a distance [1,6], Roll, pitch and yaw refer to rotations about the respective axes starting from a defined equilibrium state. The equilibrium roll angle is known as wings level or zero bank angle, equivalent to a level heeling angle on a ship. Yaw is known as "heading". The equilibrium pitch angle in submarine and airship parlance is known as "trim".

\subsection{Longitudinal Modes}

Oscillating motions can be described by two parameters, the period of time required for one complete oscillation, and the time required to damp to half-amplitude, or the time to double the amplitude for a dynamically unstable motion. The longitudinal motion consists of two distinct oscillations, a long-period oscillation called a phugoid mode and a short-period oscillation referred to as the short-period mode [7].

\subsubsection{Phugoid Oscillations}

The longer period mode, called the "phugoid mode" is the one in which there is a largeamplitude variation of air-speed, pitch angle, and altitude, but almost no angle-of-attack variation. The phugoid oscillation is really a slow interchange of kinetic energy (velocity) and potential energy (height) about some equilibrium energy level as the aircraft attempts to reestablish the equilibrium level-flight condition from which it had been disturbed. The motion is so slow that the effects of inertia forces and damping forces are very low. Although the damping is very weak, the period is so long that the pilot usually corrects for this motion without being aware that the oscillation even exists. Typically the period is $20-60$ seconds $[3,11]$.

\subsubsection{Phugoid Short period oscillation}

With no special name, the shorter period mode is called simply the "short-period mode". The short-period mode is a usually heavily damped oscillation with a period of only a few seconds. The motion is a rapid pitching of the aircraft about the center of gravity. The period is so short that the speed does not have time to change, so the oscillation is essentially an angleof-attack variation. The time to damp the amplitude to one-half of its value is usually on the order of 1 second. Ability to quickly self damp when the stick is briefly displaced is one of the many criteria for general aircraft certification [4]. 


\section{Aircraft Longitudinal Dynamics}

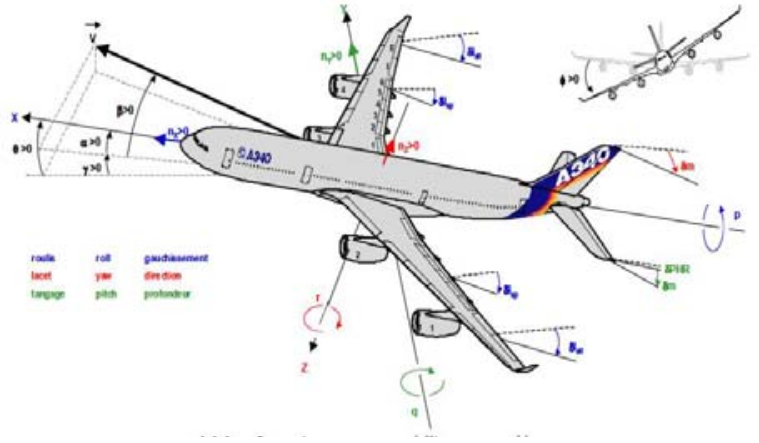

Figure 2. Aerodynamic reference

\subsection{Equations of Movements}

Equations of the movement are governed by the equations of mechanics

$$
\begin{aligned}
& \left\{\begin{array}{l}
m \frac{\overrightarrow{d u}}{d t}=\sum \overrightarrow{F_{e}} \\
\frac{\overrightarrow{d C}}{d t}=\sum \overrightarrow{M_{e}}
\end{array}\right. \\
& \beta=p=r=\Phi=0
\end{aligned}
$$

Longitudinal equations can be rewritten as:

$$
\left\{\begin{aligned}
\dot{u}= & \frac{\mathrm{X}_{\mathrm{u}}}{m} u+\frac{\mathrm{X}_{\mathrm{w}}}{m} w-\frac{g \cos \Theta_{0}}{m} \theta+\Delta \mathrm{X}^{\mathrm{c}} \\
\dot{w}= & \frac{Z_{u}}{m-Z_{\dot{w}}} u+\frac{Z_{w}}{m-Z_{\dot{w}}} w+\frac{Z_{q+m U_{0}}}{m-Z_{\dot{w}}} q-\frac{m g \sin \Theta_{0}}{m-Z_{\dot{w}}} \theta+\Delta Z^{c} \\
\dot{q}= & \frac{\left[M_{u}+Z_{u} \Gamma\right]}{I_{y y}} u+\frac{\left[M_{u}+Z_{u} \Gamma\right]}{I_{y y}} w+\frac{\left[M_{q}+\left(z_{q}+m U_{0}\right) \Gamma\right]}{I_{y y}} \\
& -\frac{m g \sin \Theta_{0} \Gamma}{I_{y y}} \theta+\Delta M^{c} \\
\dot{\theta}= & q
\end{aligned}\right.
$$

With:

$$
\begin{aligned}
\Delta X^{c} & =\frac{X_{\delta_{e}}}{m} \delta_{e}+\frac{X_{p}}{m} \delta_{p} \\
\Delta Z^{c} & =\frac{Z_{\delta_{e}}}{m-Z_{\dot{\omega}}} \delta_{e}+\frac{Z_{\delta_{P}}}{m-Z_{\dot{\omega}}} \delta_{p} \Delta M^{c}=\frac{M_{\delta_{e}}+Z_{\delta_{e}} \Gamma}{I_{y y}} \delta_{e}+\frac{M_{\delta_{p}}+Z_{\delta_{p}}\ulcorner}{I_{y y}} \delta_{p}
\end{aligned}
$$

Rewrite in state space form as:

Since $u \approx 0$ in this mode, then $\dot{u} \approx 0$ and can eliminate the $\mathrm{X}$ force equation:

$$
\begin{aligned}
& {\left[\begin{array}{c}
\dot{W} \\
\dot{q} \\
\dot{\theta}
\end{array}\right]=\left[\begin{array}{ccc}
\frac{Z_{w}}{m-Z_{\dot{w}}} & \frac{Z_{q}+m U_{0}}{m-Z_{\dot{w}}} & \frac{-m g \sin \Theta_{0}}{m-Z_{\dot{w}}} \\
\frac{\left[M_{w}+Z_{w} \Gamma\right]}{I_{y y}} & \frac{\left[M_{q}+\left(z_{q}+m U_{0}\right) \Gamma\right]}{I_{y y}} & \frac{-m g \sin \Theta_{0}}{I_{y y}} \\
0 & 1 & 0
\end{array}\right]\left[\begin{array}{c}
W \\
q \\
\theta
\end{array}\right]+\left[\begin{array}{c}
\Delta Z^{c} \\
\Delta \mathrm{M}^{\mathrm{c}} \\
0
\end{array}\right]} \\
& {\left[\begin{array}{c}
\dot{W} \\
\dot{q} \\
\dot{\theta}
\end{array}\right]=\left[\begin{array}{ccc}
\frac{Z_{w}}{m} & U_{0} & -g \sin \Theta_{0} \\
\frac{\left[M_{w}+Z_{w} \frac{\mathrm{M}_{\dot{w}}}{\mathrm{~m}}\right]}{I_{y y}} & \frac{\left[M_{q}+\left(m U_{0}\right) \frac{\mathrm{M}_{\dot{\mathrm{w}}}}{\mathrm{m}}\right]}{I_{y y}} & \frac{-m g \sin \Theta_{0}}{I_{y y}} \frac{M_{\dot{w}}}{m} \\
0 & 1 & 0
\end{array}\right]\left[\begin{array}{c}
W \\
q \\
\theta
\end{array}\right]+\left[\begin{array}{c}
\Delta Z^{c} \\
\Delta \mathrm{M}^{\mathrm{c}} \\
0
\end{array}\right]}
\end{aligned}
$$


The transfer function can be represented in state-space form and output equation as state by equation:

$$
\begin{aligned}
& {\left[\begin{array}{c}
\dot{w} \\
\dot{q} \\
\dot{\theta}
\end{array}\right]=\left[\begin{array}{ccc}
-0.3149 & 235.8928 & 0 \\
-0.0034 & -0.4282 & 0 \\
0 & 1 & 0
\end{array}\right]\left[\begin{array}{l}
w \\
q \\
\theta
\end{array}\right]+\left[\begin{array}{c}
-5.5079 \\
0.0021 \\
0
\end{array}\right] \delta_{e}} \\
& y=\left[\begin{array}{lll}
0 & 0 & 1
\end{array}\right]\left[\begin{array}{l}
w \\
q \\
\theta
\end{array}\right]+[0]
\end{aligned}
$$

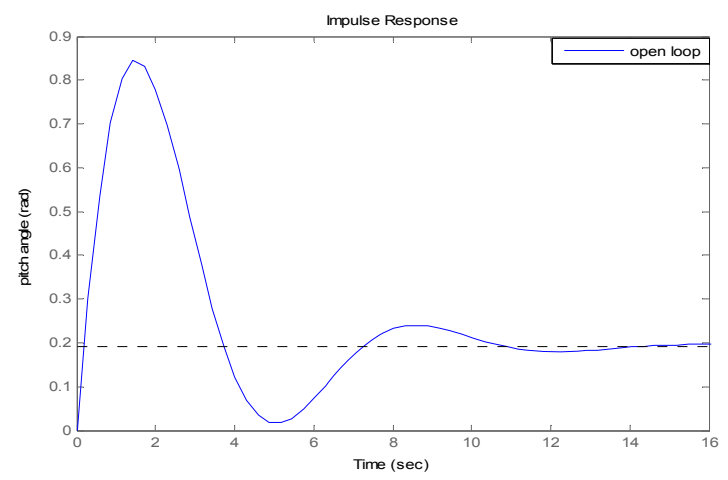

Figure 3. Open loop Impulse Response (Pitch angle)

$X=[u, \omega, q, \gamma]^{T}$ and $\gamma=\theta-\alpha$ represent flight path angle, with $\alpha=\omega, u=\left[\begin{array}{l}\delta_{e} \\ \delta_{p}\end{array}\right]$

The input (elevator deflection angle, $\delta_{e}$ ) will be $0.2 \mathrm{rad}(11$ degrees), and the output is the pitch angle (theta).

$$
X=[u, \omega, q, \gamma]^{T} \text { and } \gamma=\theta-\alpha \text { represent flight path angle, with } \alpha=\omega, u=\left[\begin{array}{l}
\delta_{e} \\
\delta_{p}
\end{array}\right]
$$

The input (elevator deflection angle, $\delta_{e}$ ) will be 0.2 rad (11 degrees), and the output is the pitch angle (theta).

There are three types of possible lateral-directional dynamic motion: roll subsidence mode, Dutch roll mode, and spiral mode.

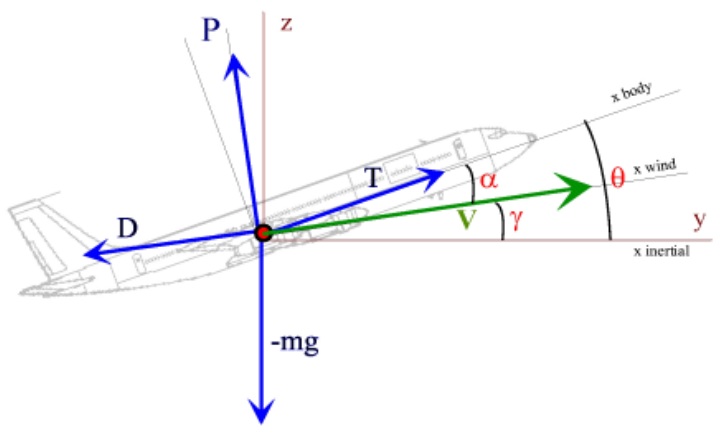

Figure 4. Definition of forces, moments and angles

\section{Aircraft Lateral Dynamics}

There are three types of possible lateral-directional dynamic motion: roll subsidence mode, Dutch roll mode, and spiral mode. 


\section{1, Roll Subsidence Mode}

Roll subsidence mode is simply the damping of rolling motion. There is no direct aerodynamic moment created tending to directly restore wings-level, i.e. there is no returning "spring force/moment" proportional to roll angle. However, there is a damping moment (proportional to roll rate) created by the slewing-about of long wings. This prevents large roll rates from building up when roll-control inputs are made or it damps the roll rate (not the angle) to zero when there are no roll-control inputs. Roll mode can be improved by adding dihedral effects to the aircraft design, such as high wings, dihedral angles or sweep angles.

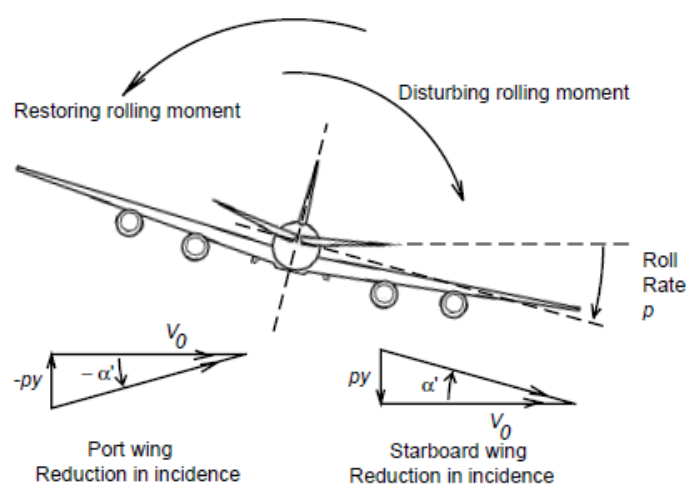

Figure 5. Roll subsidence mode

Using a procedure similar to the longitudinal mode, we can develop the equation of motion for the lateral dynamics.

$$
\dot{x}=A x+B u, x=\left[\begin{array}{l}
\beta \\
p \\
r \\
\phi
\end{array}\right], u=\left[\begin{array}{l}
\delta_{a} \\
\delta_{r}
\end{array}\right]
$$

$x^{T}=[\beta p r \phi]^{T}:$ state vector

$u^{T}=\left[\delta_{a} \delta_{r}\right]^{T}$ : control vector

$\delta_{a}, \delta_{r}$ : aileron and rudder deflection

$\beta, \phi$ : sideslip and roll angle

$p, r$ : roll and yaw rate

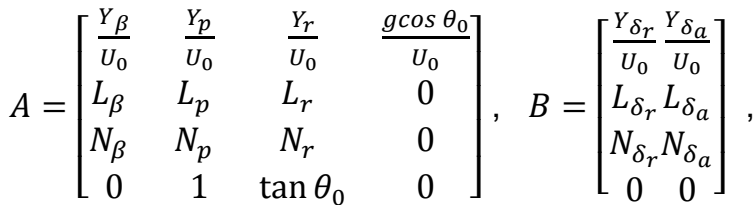

$$
\begin{aligned}
& C=\left[\begin{array}{llll}
1 & 0 & 0 & 0 \\
0 & 0 & 0 & 1
\end{array}\right], \quad D=\left[\begin{array}{ll}
0 & 0 \\
0 & 0
\end{array}\right]
\end{aligned}
$$

If we assume that the measurable outputs are the sideslip angle $\beta$ and roll angle $\phi$, the matrixes $A, B$ and $C$ are:

$$
A=\left[\begin{array}{cccc}
-0.0558 & -0.9968 & 0.0802 & 0.0415 \\
0.5980 & -0.1150 & -0.0318 & 0 \\
-3.0500 & 0.3880 & -0.4650 & 0 \\
0 & 0.0805 & 1.000 & 0
\end{array}\right],
$$




$$
B=\left[\begin{array}{cl}
0.0729 & 0.000 \\
-4.7500 & 0.00775 \\
0.15300 & 0.1430 \\
0 & 0
\end{array}\right], \quad C=\left[\begin{array}{llll}
1 & 0 & 0 & 0 \\
0 & 0 & 0 & 1
\end{array}\right]
$$

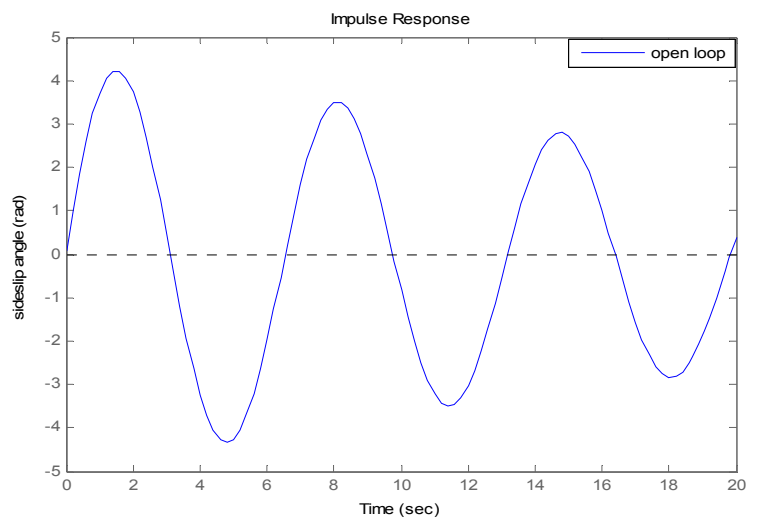

Figure 6. Open loop Impulse Response (Sideslip angle)

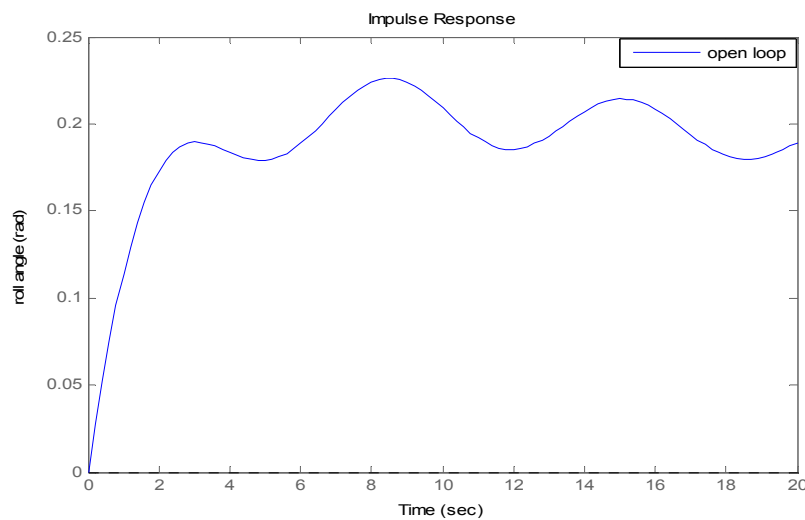

Figure 7. Open loop Impulse Response (Roll angle)

\subsection{Spiral Mode}

If a spirally unstable aircraft, through the action of a gust or other disturbance, gets a small initial roll angle to the right, for example, a gentle sideslip to the right is produced. The sideslip causes a yawing moment to the right. If the dihedral stability is low, and yaw damping is small, the directional stability keeps turning the aircraft while the continuing bank angle maintains the sideslip and the yaw angle. This spiral gets continuously steeper and tighter until finally, if the motion is not checked a steep, high-speed, spiral dive results. The motion develops so gradually, however that it is usually corrected unconsciously by the pilot, who may not be aware that spiral instability exists. If the pilot cannot see the horizon, for instance because of clouds, he might not notice that he is slowly going into the spiral dive, which can lead into the graveyard spiral. To be spirally stable, an aircraft must have some combination of a sufficiently large dihedral, which increases roll stability, and a sufficiently long vertical tail arm, which increases yaw damping. Increasing the vertical tail area then magnifies the degree of stability or instability. The spiral dive should not be confused with a spin. 


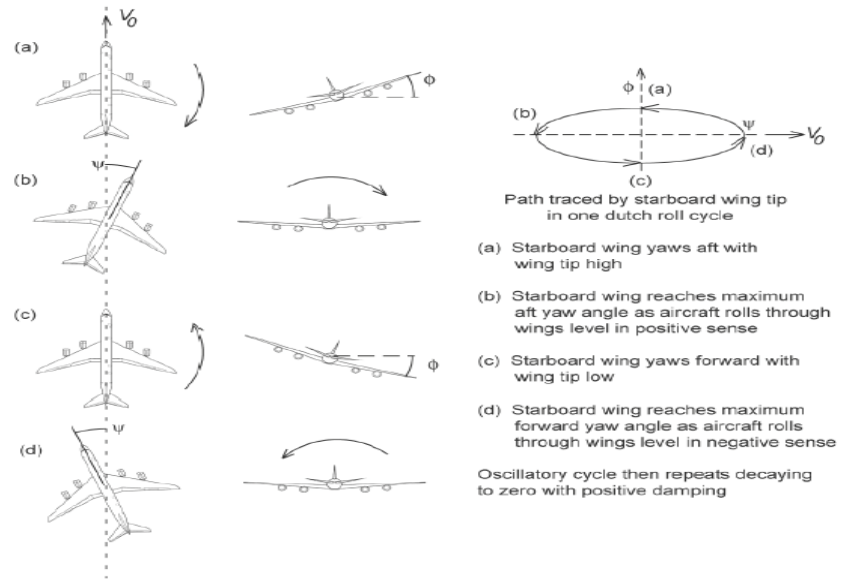

Figure 8. Spiral mode

\subsection{Dutch Roll}

The second lateral motion is an oscillatory combined roll and yaw motion called Dutch roll, perhaps because of its similarity to an ice-skating motion of the same name made by Dutch skaters; the origin of the name is unclear. The Dutch roll may be described as a yaw and roll to the right, followed by a recovery towards the equilibrium condition, then an overshooting of this condition and a yaw and roll to the left, then back past the equilibrium attitude, and so on. The period is usually on the order of 3-15 seconds, but it can vary from a few seconds for light aircraft to a minute or more for airliners. Damping is increased by large directional stability and small dihedral and decreased by small directional stability and large dihedral. Although usually stable in a normal aircraft, the motion may be so slightly damped that the effect is very unpleasant and undesirable. In swept-back wing aircraft, the Dutch roll is solved by installing a yaw damper, in effect a special-purpose automatic pilot that damps out any yawing oscillation by applying rudder corrections. Some swept-wing aircraft have an unstable Dutch roll. If the Dutch roll is very lightly damped or unstable, the yaw damper becomes a safety requirement, rather than a pilot and passenger convenience. Dual yaw dampers are required and a failed yaw damper is cause for limiting flight to low altitudes, and possibly lower match numbers, where the Dutch roll stability is improved.

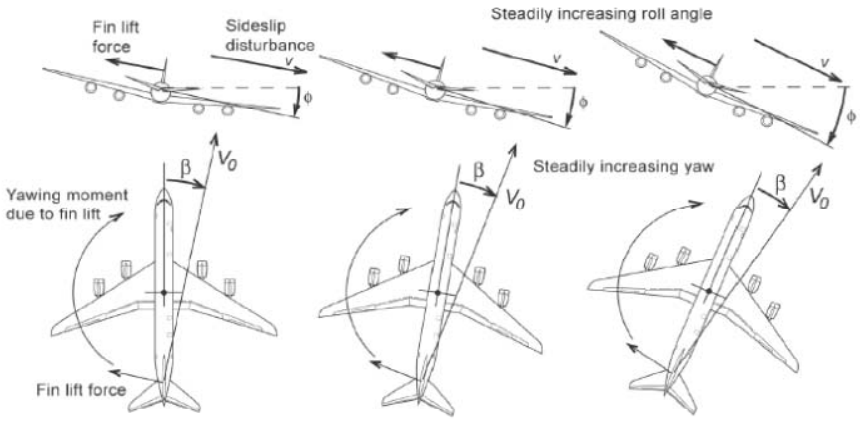

Figure 8. Dutch roll mode

From examining Figure 6, it can be seen that dynamical behavior of an aircraft is not acceptable considering overshoot, rise time, settling time and steady-state error values, and must be modified using feedback control 


\section{Model Predictive Controller}

\subsection{The Receding Horizon Idea}

The figure shows the basic idea of predictive control. In this presentation of the basics, we confine ourselves to discussing the control of a single-input, single-output (SISO) plant. We assume a discrete-time setting, and that the current time is labeled as time step k.at the current time the plant output is $y(k)$, and that the figure shows the previous history of the output trajectory. Also shown is a set point trajectory, which is the trajectory that the output should follow, ideally. The value of the set-point trajectory at any time $t$ is denoted by $s(t)$.

Distinct from the set-point trajectory is the reference trajectory. This starts at the current output $y(k)$, and defines an ideal trajectory along which the plant should return to the set-point trajectory, for instance after a disturbance occurs. The reference trajectory therefore defines an important aspect of the closed-loop behaviour of the controlled plant. It is not necessary to insist that the plant should be driven back to the set-point trajectory as fast as possible, although that choice remains open. It is frequently assumed that the reference trajectory as fast as possible, although that choice remains open. It is frequently assumed that the reference trajectory approaches the set point exponentially, which we shall denote Tref, defining the speed of response. That is the current error is

$$
e(k)=s(k)-y(k)
$$

Then the reference trajectory is chosen such that the error $i$ steps later, if the output followed it exactly, would be:

$$
\begin{aligned}
e(k+1) & =\exp (-i T s / \text { Tref }) * e(k) \\
& =\lambda i * e(k)
\end{aligned}
$$

Where $T s$ is the sampling interval and $\lambda=\exp \left(-\frac{\pi s}{\pi r a}\right)$ (note that $\left.0<\lambda<1\right)$. That is, the reference trajectory is defined to be:

$$
\begin{aligned}
r(k+i k) & =s(k+i)-\epsilon(k+i) \\
& =s(k+i)-\exp \left(-\frac{r \sigma}{\sigma}\right) * \epsilon(k)
\end{aligned}
$$

The notation $r(k+s k)$ indicates that the reference trajectory depends on the conditions at time $k$, in general. Alternative definitions of the reference trajectory are possible-For e.g., a straight line from the current output which meets the set point trajectory after a specified time. follows:

Summarizing the main steps involved in implementing DMC on a process are as

1. Develop a discrete step response model with length $\mathrm{N}$ based on sample time $\mathrm{t}$

2. Specify the prediction $(P)$ and control $(M)$ horizons. $N \geq P \geq M$

3. Specify the weighting on the control action ( $w=0$ if no weighting on the control action ( $w=0$ if no weighting).

4. All calculations assume deviation variable form, so remember to convert to/from physical units.

The effect of all these tuning parameters is now discussed for SISO systems.

Model-length and sample-time selection are independent. The model length should be approximately the 'settling time' of the process, that is, the time required to reach a new steady state after a step input change. For most systems, the model length is roughly 50 coefficients. The sample time is usually on the order of one tenth the dominant time constant, so the model length is roughly the settling time of the process.

Prediction and control horizons differ in length. Usually, the prediction horizon is selected to be much longer than the control horizon. This is particularly true if the control weighting factor is selected to be zero. Usually, if the prediction horizon is much longer than the control horizon, the control system is less sensitive to model error. Often $\mathrm{P}=20$ or so, while $M=1-3$. 
Control weighting is often step to zero if the prediction horizon is much longer the control horizon. As the control horizon is increased, the control moves tend to become more aggressive so larger weight is needed to penalize the control moves.

\subsection{Objective Functions}

Here, there are several different choices for objectives functions. The first one that comes to mind is a standard least-squares or "quadratic "objective function. The objective function is a "sum of squares "of the predicted errors (differences between the set points and model-predicted outputs) and the control moves (changes in control action from step to step). A quadratic objective function for a prediction horizon of 3 and a control horizon of 2 can be written.

$$
\begin{aligned}
& \Phi=(R k+1-\hat{y} k+1)^{\wedge} 2+(R k+2-\hat{y} k+2)^{\wedge} 2+(R k+3-\hat{y} k+3)^{\wedge} 2+w \Delta U k^{\wedge} 2+ \\
& w \Delta U k+1^{\wedge} 2
\end{aligned}
$$

Where $\hat{y}$ represents the model predicted output, $r$ is the set point, $\Delta U$ is the change in manipulated input from one sample to the next, $w$ is a weight for the changes in the manipulated input, and the subscripts indicate the sample time ( $\mathrm{k}$ is the current sample time). For a prediction horizon of $P$ and a control horizon of $M$, the least Squares objective function is written.

$$
\Phi=\sum(R k+1-\hat{y} k+1)^{\wedge} 2+w \sum \Delta U k+1^{\wedge} 2
$$

Another possible objective function is to simply take a sum of the absolute values of the predicted errors and control moves. For a prediction horizon of 3 and a control horizon of 2, the absolute value objective function is:

$$
\begin{aligned}
& \Phi=|(R k+1-\hat{y} k+1)|+|(R k+2-\hat{y} k+2)|+|(R k+3-\hat{y} k+3)|+w|\Delta U k|+ \\
& w|\Delta U k+1|
\end{aligned}
$$
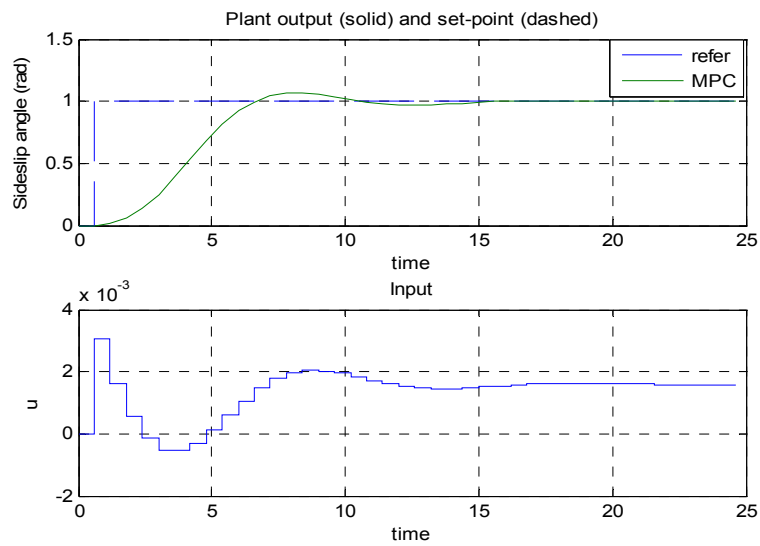

Figure 9. Sideslip angle after applying MPC to the aircraft

The controller is able to track the sideslip angle reference as long as it is feasible reference. At 10 seconds the MPC stable the sideslip angle to track a reference, and remain the rudder deflection in the initial conditions, the result is shown in Figure 9.

The predictive controller has already included it into the linear model which did help the system to provide the necessary stability to perform the rudder and with reduced variations in the sideslip angle. 

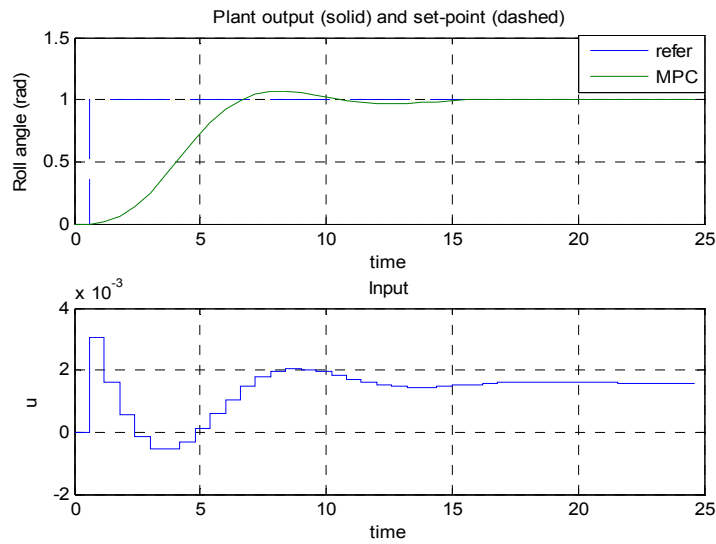

Figure 10. Roll angle after applying MPC to the aircraft

The controller is able to track the roll angle reference as long as it is feasible reference. At 10 seconds the MPC stable the sideslip angle to track a reference, and remain the aileron deflection in the initial conditions, the result is shown in Figure 10.

The predictive controller has already included it into the linear model which did help the system to provide the necessary stability to perform the aileron and with reduced variations in the roll angle.
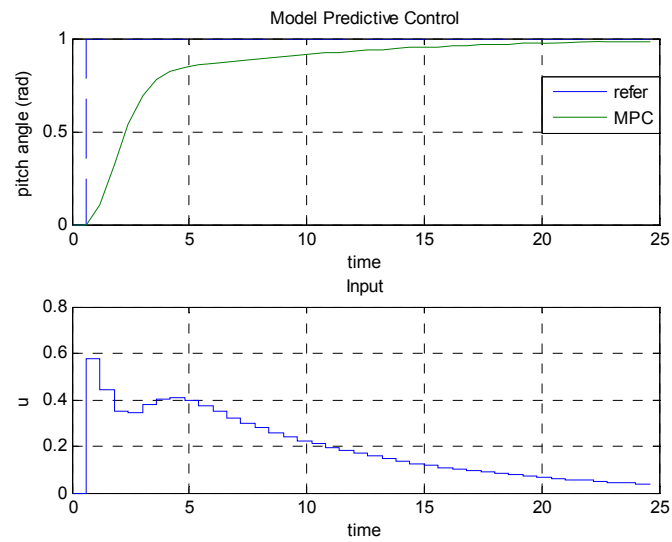

Figure 11. Pitch angle after applying MPC to the aircraft

Consequently, by tuning the value of $x=500$, the following values of matrix $K$ are obtained. If $x$ is increased even higher, improvement to the response should be obtained even more. But for this case, the values of $x=500$ is chosen because it satisfied the design requirements while keep $x$ as small as possible.

The controller is able to track the pitch angle reference as long as it is feasible reference. At 20 seconds the MPC stable the sideslip angle to track a reference, and remain the elevator deflection in the initial conditions, the result is shown in Figure 12.

The predictive controller has already included it into the linear model which did help the system to provide the necessary stability to perform the elevator and with reduced variations in the pitch angle.

We can see that the reference tracking is good, but the controller is still not sufficiently robust in certain segments. The main advantage of model predictive controller is depicted in a close up of bird perspective of the aircraft trajectory in Figure 11, where we can clearly observe a preliminary action of the controller, a direct consequence of the long predictive horizon and the look ahead function. 
Useful for obvious reasons, the look ahead function allows the controller to see the future reference and thus enables the preliminary action of the controller. The controller still has some problems, specifically it the ratio between robustness and performance is not optimal.

\section{Conclusion}

This paper presented an evaluation of a model based predictive controller used to control the aircraft pitch, sideslip and roll angle. A simplified linear model was used to simulate the system response. The predictive controller made use of a linearized state space representation in order to obtain the system predicted behaviour for optimization purposes. With the introduction of the dynamic reference generator we have been able to translate the model based predictive control algorithm into the spatial mode, meaning that the reference vectors fed to the controllers are dependent on the current aircraft position. This enables the plane to land on a specific location rather than at specific time, which is very useful for obvious reasons. The look ahead function allows the controller to see the future reference and thus enables the preliminary action of the controller. The controller still has some problems, specifically it the ratio between robustness and performance is not optimal.

\section{References}

[1] Camacho EF, Bordons C. Model Predictive Control. 2nd ed. London: Springer Control. University of Notre Dame. 2000

[2] Durham WC. Aircraft Dynamics and Control". 1st ed. Virginia: Virginia Polytechnic Institute and State University. 1997.

[3] Ebdon BW, Heise SA. Model predictive control for fault tolerance in aerospace systems. In: H. Poor. Editor. An Introduction to Signal Detection and Estimation. New York: Springer-Verlag. 1985: 4.

[4] Ferramosca A, Limon D, Alvarado I, Alamo T, Camacho E. MPC for tracking with optimal closed-loop performance. Automatica. 2009; 45(8): 1975-1978.

[5] Ji-Hong Zhu. A Survey of Advanced Flight Control Theory and Application. MACS Multi conference on Computational Engineering in System Application (CESA). China. 2006.

[6] Hamid R Berenji, Sujit Saraf, Ping-Wei Chang, Steven R Swanson. Pitch Control of the Space Shuttle Training Aircraft. IEEE Transaction on Control System Technology. 2001; 9(3).

[7] Labane C, Zemalache MK. Aircraft System Control Using LQG and LQR Controller with Optimal Estimation -Kalman Filter. Elsevier Procedia Engineering. 2001; 80: 245-257.

[8] Ling KV, Yue SP, Maciejowski JM. A fpga implementation of model predictive control. Proceedings of the American Control Conference. Minneapolis. 2006.

[9] Maciejowski JM, Heise SA. Model predictive control of a super maneuvarable aircraft. American Institute of Aeronautics and Astronautics. 1996.

[10] Maciejowski JM. Predictive Control with Constraints. 1st ed. Harlow: Prentice Hall PTR. Mathworks. 2009.

[11] Mehra RK, Prasanth RK, Bennet RL, Wasikovski M. Model predictive control design for xv-15 tilt rotor flight control. American Institute of Aeronautics and Astronautics. 2001.

[12] Miotto P, Lepome RC. Design of a model predictive control flight control system for a reusable launch vehicle. In: AIAA Guidance, Navigation, and Control Conference and Exhibit. Massachusetts. 2003.

[13] Nelson RC. Flight Stability and Automatic Control. Second Edition. McGraw Hill. 1998.

[14] Robert S Eick. A Reconfiguration Scheme for Flight Control Adaptation to Fixed Position Actuator Failures. Ph. D Theses. University of Florida. 2003.

[15] Stojiljkovic B Vasov, L Mitrovic, C Cvetkovic D. The Application of the Root Locus Method for the Design of Pitch Controller of an F-104A Aircraft. Journal of Mechanical Engineering. 2009; 55.

[16] Simon D, öfberg J, Glad T. Reference tracking MPC using terminal set scaling. In 51st IEEE Conference on Decision and Control (CDC). 2012: 4543-4548.

[17] Simon D, Löfberg J, Glad T. Nonlinear Model Predictive Control using Feedback Linearization and Local Inner Convex Constraint Approximations. In Proceedings of the 2013 European Control Conference. 2013; 3: 2056-2061.

[18] Thomas J Redling. Integrated Flight Control System; A New Paradigm for an Old Art. IEEE Aerospace and Electronic Systems Society (AESS) Systems Magazine. 2001. 